\title{
Magnetization-induced second-harmonic generation of light by exchange-coupled magnetic layers
}

\author{
L. C. Sampaio, ${ }^{*}$ J. Hamrle, ${ }^{\dagger}$ V. V. Pavlov, ${ }^{\ddagger}$ and J. Ferré \\ Laboratoire de Physique des Solides, Unité Mixte de Recherche 8502, Centre National de la Recherche Scientifique, \\ Bat. 510, Université Paris-Sud, 91405 Orsay, France \\ P. Georges and A. Brun \\ Institut d'Optique Théorique et Appliquée, Unité Mixte de Recherche 8501, Centre National de la Recherche \\ Scientifique, Bat. 503, Université Paris-Sud, 91405 Orsay, France \\ H. Le Gall and J. Ben Youssef \\ Laboratoire de Magnétisme de Bretagne, Centre National de la Recherche Scientifique, Université de Brest, \\ 29285 Brest, France
}

Received May 26, 2004; accepted August 7, 2004; revised manuscript received September 6, 2004

\begin{abstract}
A longitudinal magneto-optical Kerr effect and magnetization-induced second-harmonic generation (MSHG) of light $\left(\right.$ at $2 \omega$ ) have been measured in a $\mathrm{SiO}_{2} / \mathrm{Fe}_{96} \mathrm{Si}_{4} / \mathrm{Dy}_{30} \mathrm{Fe}_{58} \mathrm{Co}_{12} /$ glass exchange-coupled magnetic bilayer system with competitive anisotropies. Theoretical MSHG predictions in this structure that give rise to an effect proportional to magnetization components and that are allowed by an electric dipole mechanism are reported and discussed. The magnitude of the MSHG effect depends on the electric field of the incoming radiation at each interface and on the corresponding incoming (at $\omega$ ) and outgoing (at $2 \omega$ ) Fresnel coefficients. It is demonstrated that transverse $p p$ MSHG selectively probes the magnetization of the first $\mathrm{SiO}_{2} / \mathrm{Fe}_{96} \mathrm{Si}_{4}$ interface, while transverse $s p$ and longitudinal $p$ s MSHG is sensitive, but less selectively, to the $\mathrm{Fe}_{96} \mathrm{Si}_{4} / \mathrm{Dy}_{30} \mathrm{Fe}_{58} \mathrm{Co}_{12}$ interface that supports a planar magnetic domain wall. ( $p$ and $\mathrm{s}$ are the usual parallel and perpendicular polarizations to the plane of incidence.) The contribution to MSHG by gradient magnetization terms is negligible. (C) 2005 Optical Society of America
\end{abstract}

OCIS codes: $160.3820,160.4330,190.4350,230.4110$.

\section{INTRODUCTION}

The magneto-optical Kerr effect (MOKE) has been used to probe the magnetic properties of thin-layered and multilayered structures. ${ }^{1}$ It gives information on the magnetic state of the structure over the light's penetration depth, i.e., a few tens of nanometers in metals. Magnetic surface properties are now well probed by electron techniques such as spin-polarized scanning-electron microscopy, ${ }^{2}$ spin-polarized low-energy electron microscopy, ${ }^{3}$ and spin-polarized scanning tunneling microscopy. ${ }^{4}$ So most unsolved problems concern buried interface magnetism in multilayer structures. There are few available techniques to check interface magnetism, and the usefulness of magnetization-induced secondharmonic generation (MSHG) for this purpose has still to be confirmed. For electric dipole mechanisms, which are often favored in centrosymmetric thin metallic film structures, MSHG is predicted to come only from the surface and interfaces as a consequence of local symmetry breaking. This interface-induced effect has been predicted theoretically, and light-polarization selection rules have been deduced for all-optical configurations. ${ }^{5,6}$ Whereas it has been demonstrated experimentally that MSHG undoubtedly comes from surface magnetism, ${ }^{7-10}$ there have been few studies of real multilayer structures involving several types of buried interface. ${ }^{11-15}$

Our aim in the present study is to check theoretical predictions about MSHG in a simple exchange-coupled layer structure built from two magnetic layers in contact that have perpendicular and in-plane anisotropy, respectively. MSHG is then assumed to check partially the magnetization state at their interface, on which is centered a planar domain wall. A search for MSHG contributions that are due to magnetization gradients ${ }^{16}$ can be also initiated in that case.

Such a type of structure is commonly used in the design of magneto-optical and magnetic recording media and for achieving new generations of magnetic memories. In thermally assisted writing of the information, such a structure has been proposed to improve the direct overwriting process ${ }^{17}$ and to design a recording-readout stack of layers for achieving magnetic superesolution. ${ }^{18}$ In perpendicular recording, a capping layer with in-plane anisotropy favors magnetic flux closure and thus lowers the write-erase field. ${ }^{19}$ For coupled ferrimagneticferromagnetic bilayers, as studied here, an exchange bias field acts on the soft ferromagnetic layer with in-plane anisotropy. ${ }^{20}$ This kind of exchange-coupled bilayer 
structure is also needed for stabilizing the magnetization in the hard layer of magnetic random-access memories.

\section{EXPERIMENT}

A ternary rare-earth transition metal $\mathrm{Dy}_{30} \mathrm{Fe}_{58} \mathrm{Co}_{12}$ layer was grown on a 1-mm-thick glass substrate by sputtering of the elements at an Ar pressure of $1.5 \times 10^{-3}$ Torr at a deposition rate of $9.3 \mathrm{~nm} / \mathrm{min}$. Above this layer a $\mathrm{Fe}_{96} \mathrm{Si}_{4}$ layer was subsequently grown by sputtering at 7.5 $\times 10^{-3}$-Torr Ar pressure at a deposition rate of $4 \mathrm{~nm} /$ min. Finally, a $10-\mathrm{nm}$-thick $\mathrm{SiO}_{2}$ layer was deposited on the top of this structure to prevent its oxidation. This structure (Fig. 1) can serve as a magnetic or magnetooptic recording medium. Two samples were prepared. In each case the $\mathrm{Dy}_{30} \mathrm{Fe}_{58} \mathrm{Co}_{12}$ layer thickness was fixed at $30 \mathrm{~nm}$, but the FeSi layer was either 5 or $10 \mathrm{~nm}$ thick. $\mathrm{X}$-ray analyses have confirmed the amorphous character of both the $\mathrm{Dy}_{30} \mathrm{Fe}_{58} \mathrm{Co}_{12}$ and the $\mathrm{Fe}_{96} \mathrm{Si}_{4}$ layers. We also prepared two other $\mathrm{Dy}_{30} \mathrm{Fe}_{58} \mathrm{Co}_{12}$ or $\mathrm{Fe}_{96} \mathrm{Si}_{4}$ single-layer samples to check independently their magnetic and magneto-optical properties.

The composition of the ferrimagnetic $\mathrm{Dy}_{30} \mathrm{Fe}_{58} \mathrm{Co}_{12}$ layer was selected to produce a large uniaxial anisotropy constant $K_{1}$ (equal to $1.710^{6} \mathrm{erg} / \mathrm{cm}^{3}$ at room temperature) favoring an out-of plane orientation of both rareearth and transition metal sublattice magnetization. As we shall see below, the temperature of the sample was raised to $80^{\circ} \mathrm{C}$ under focused laser irradiation. So the $\mathrm{Dy}_{30} \mathrm{Fe}_{58} \mathrm{Co}_{12}$ layer composition was also chosen to produce a compensation temperature $\left(T_{\text {comp }}=75^{\circ} \mathrm{C}\right)$ close to that reached during measurements. This layer also exhibits a low Curie temperature, $T_{c} \approx 180^{\circ} \mathrm{C}$. Under these conditions the saturated magnetization of the illuminated $\mathrm{Dy}_{30} \mathrm{Fe}_{58} \mathrm{Co}_{12}$ layer becomes much lower than its room-temperature value. Thus the interface exchange interaction is reduced sufficiently to permit the planar wall to be located easily in the vicinity of the $\mathrm{Dy}_{30} \mathrm{Fe}_{58} \mathrm{Co}_{12} / \mathrm{Fe}_{96} \mathrm{Si}_{4}$ interface. The composition of the ferromagnetic $\mathrm{Fe}_{96} \mathrm{Si}_{4}$ layer was selected to produce a weak in-plane anisotropy and a small coercive field. The room-temperature out-of-plane and in-plane coercivity of single $\mathrm{Dy}_{30} \mathrm{Fe}_{58} \mathrm{Co}_{12}$ and $\mathrm{Fe}_{96} \mathrm{Si}_{4}$ layers, measured by the longitudinal magneto-optical Kerr effect (LMOKE), was

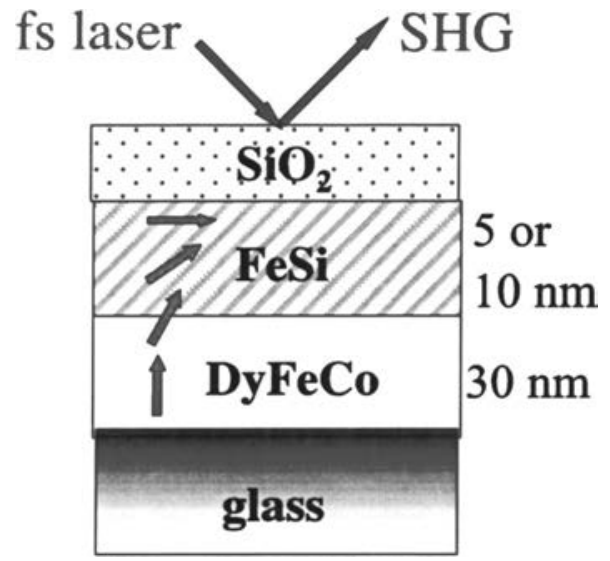

Fig. 1. Sample structure: fs, femtosecond; SHG, secondharmonic generation.

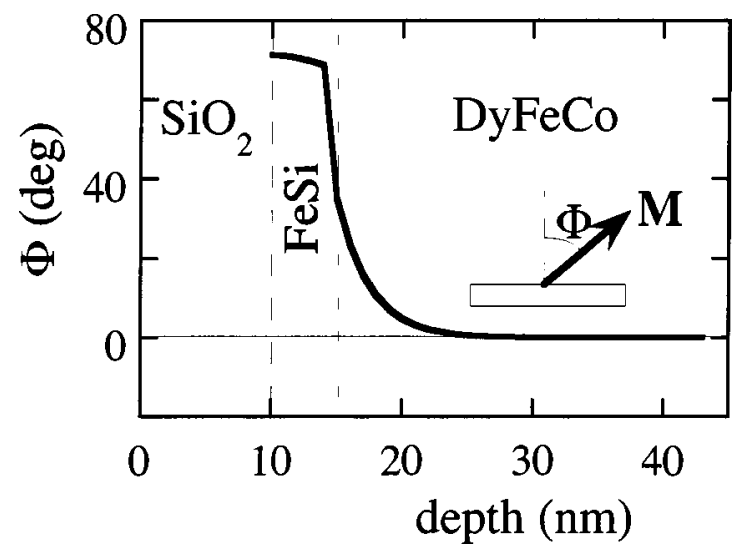

Fig. 2. In-depth variation of deviation angle of magnetization $\Phi$ with respect to the film normal. The depth was measured from the air- $\mathrm{SiO}_{2}$ interface.

$2.5 \mathrm{kOe}$ and $3 \mathrm{Oe}$, respectively. The room-temperature saturated magnetization of $\mathrm{Fe}_{96} \mathrm{Si}_{4}$ was estimated to be $1300 \mathrm{emu} / \mathrm{cm}^{3}$.

In the exchange coupled layer structure the competition between in-plane and out-of plane anisotropies is efficient mainly at the $\mathrm{Dy}_{30} \mathrm{Fe}_{58} \mathrm{Co}_{12} / \mathrm{Fe}_{96} \mathrm{Si}_{4}$ interface. The $\mathrm{Dy}_{30} \mathrm{Fe}_{58} \mathrm{Co}_{12}$ layer still shows a square loop in a perpendicular field but with a smaller coercivity $\left(H_{c}\right.$ $=1.3 \mathrm{kOe}$ at room temperature) than for the same isolated film. An exchange bias effect is then expected between the ferrimagnetic and ferromagnetic layers, ${ }^{20}$ but for our sample cooled in zero field through the Curie temperature of $\mathrm{Dy}_{30} \mathrm{Fe}_{58} \mathrm{Co}_{12}$ there is no exchange bias effect. So, under that condition, the absence of any shift in the hysteresis loop above room temperature can be checked by the LMOKE. The magnetic coercivity of the $\mathrm{Fe}_{96} \mathrm{Si}_{4}$ layer, however, is nearly isotropic in the film plane. As a consequence of the exchange interaction between these magnetic layers, the room-temperature coercivity of $\mathrm{Fe}_{96} \mathrm{Si}_{4}$ is larger $\left[H_{c}=40 \mathrm{Oe}\right.$ for $\mathrm{Fe}_{96} \mathrm{Si}_{4}(5 \mathrm{~nm})$ and $52 \mathrm{Oe}$ for $\left.\mathrm{Fe}_{96} \mathrm{Si}_{4}(10 \mathrm{~nm})\right]$ than that found for the single layer $\left(H_{c} \approx 3 \mathrm{Oe}\right)$. The amorphous nature of the $\mathrm{Fe}_{96} \mathrm{Si}_{4}$ layer and the presence of a planar domain wall at the $\mathrm{Dy}_{30} \mathrm{Fe}_{58} \mathrm{Co}_{12} / \mathrm{Fe}_{96} \mathrm{Si}_{4}$ interface explain why the roomtemperature LMOKE hysteresis loop has square shape.

The competition between anisotropy orientations induces a variable deviation angle $\Phi$, with respect to the film normal, of the magnetization through the film thickness. A numerical simulation of $\Phi$ versus in-depth position $z$ from the $\mathrm{Dy}_{30} \mathrm{Fe}_{58} \mathrm{Co}_{12}$ /glass interface was simulated by the OOMMF software program. ${ }^{21}$ The following parameters $^{22}$ were used: $M_{S}=8 \mathrm{emu} / \mathrm{cm}^{3}$ for the $\mathrm{Fe}_{96} \mathrm{Si}_{4}$ layer; $K=0$ and $K_{1}=1.7 \times 10^{6} \mathrm{erg} / \mathrm{cm}^{2}$ for the $\mathrm{Fe}_{96} \mathrm{Si}_{4}$ and $\mathrm{Dy}_{30} \mathrm{Fe}_{58} \mathrm{Co}_{12}$ layers, respectively; $J=12$ $\times 10^{-12} \mathrm{erg} / \mathrm{cm}^{2}$ and $J^{\prime}=13 \times 10^{-11} \mathrm{erg} / \mathrm{cm}^{2}$ for the exchange interaction in the $\mathrm{Dy}_{30} \mathrm{Fe}_{58} \mathrm{Co}_{12}$ and the $\mathrm{Fe}_{96} \mathrm{Si}_{4}$ layers, respectively; and $J_{\text {int }}=J^{\prime} / 2$ for exchange interaction $J_{\text {int }}$ between the two magnetic layers. Whereas $\Phi$ vanishes at the $\mathrm{Dy}_{30} \mathrm{Fe}_{58} \mathrm{Co}_{12}$ /glass interface, it increases near the $\mathrm{Dy}_{30} \mathrm{Fe}_{58} \mathrm{Co}_{12} / \mathrm{Fe}_{96} \mathrm{Si}_{4}$ (5-nm) interface (Fig. 2). This means that the planar domain wall spreads inside the two magnetic layers. As a consequence, the magnetic domain wall's width is much greater than that of the in- 
termixed structural region at the interface. Thus the symmetry breaking at the interface is essentially structural in origin. The origin of MSHG at interfaces is consequently associated with efficient nonmagnetic nonlinear susceptibility terms. In a zero in-plane magnetic field the value of $\Phi$ inside the $\mathrm{Fe}_{96} \mathrm{Si}_{4}$ layer rapidly becomes nearly constant. In spite of the in-plane anisotropy character of the $\mathrm{Fe}_{96} \mathrm{Si}_{4}$ top layer, $\Phi$ does not reach $90^{\circ}$ at the $\mathrm{Fe}_{96} \mathrm{Si}_{4} / \mathrm{SiO}_{2}$ interface. The variation of $\Phi$ with the depth position for the $\mathrm{Fe}_{96} \mathrm{Si}_{4}$ (10-nm) film is quite similar to that represented in Fig. 2 for the $\mathrm{Fe}_{96} \mathrm{Si}_{4}$ (5-nm) film. In the presence of an in-plane applied field the value of $\Phi$ is increased by $\sim 15^{\circ}$ and $\sim 16^{\circ}$ in the $\mathrm{Fe}_{96} \mathrm{Si}_{4}$ layer between $H=0$ and 200 Oe for the 5- and 10 -nm-thick $\mathrm{Fe}_{96} \mathrm{Si}_{4}$ layers, respectively.

Our MSHG setup was described previously. ${ }^{15,23}$ It allows experiments to be performed in $p p, s s, p s$, and $s p$ optical configurations ${ }^{6}$ to check the components of the magnetization in the film plane $\left(M_{x}\right.$ and $M_{y}$ in the transverse or longitudinal case, i.e., for $\mathbf{H} \| \mathbf{x}$ or $\mathbf{H} \| \mathbf{y}$, respectively). The light source was a mode-locked Ti:sapphire laser that provided 100-fs pulses with 800-nm wavelength, and the MSHG signal was detected at the firstharmonic frequency, i.e., at $400 \mathrm{~nm}$. The average light power on the sample surface was $25 \mathrm{~mW}$, and the beam was focused over a $30-40-\mu \mathrm{m}$ wide spot. The LMOKE was measured with exactly the same incident light-beam conditions, but detection was achieved for light reflected at the fundamental frequency (at $800 \mathrm{~nm}$ ). Thus, all reported LMOKE and MSHG experiments were performed with the same laser beam focused at a given position on the film surface, which allowed us to compare all the data.

\section{MSHG AND FRESNEL ELEMENT CALCULATIONS}

As was discussed earlier, ${ }^{24}$ one can use either of two equivalent models for treating MSHG emission from uniformly magnetized surfaces and buried interfaces. ${ }^{24,25}$ To be consistent with our previous research we prefer to analyze our MSHG data by using the electric point dipole model. ${ }^{24}$ The present analysis does not consider higherorder terms in the expression of the dipole moment, such as that which is quadratic in magnetization, or magnetization gradients. Let us recall briefly the steps in the MSHG calculations: (i) Electric field $\mathbf{E}_{v}{ }^{(\omega)}$ at fundamental frequency $\omega$, on each interface $v$, is expressed in matrix form:

$$
\mathbf{E}_{v}{ }^{(\omega)}=\mathbf{X}_{v}{ }^{(\omega)} \mathbf{J}_{0}{ }^{(\omega)},
$$

where $\mathbf{X}_{v}^{(\omega)}$ is the matrix of the incoming generalized Fresnel coefficients and $\mathbf{J}_{0}{ }^{(\omega)}$ gives the components of the incident electric field. The $z$ axis is oriented along the normal of the film plane, and $y$ lies in the planes both of the film and of incidence.

(ii) Electric field $\mathbf{E}_{v}{ }^{(\omega)}$ gives rise to electric point dipoles oscillating on interfaces at frequency $\omega$, with moment amplitude ${ }^{24}$

$$
\boldsymbol{\mu}_{v}^{(2 \omega)}=\chi_{v} \otimes \mathbf{E}_{v}^{(\omega)} \mathbf{E}_{v}^{(\omega)},
$$

where $\chi_{v}$ is a third-rank nonlinear susceptibility tensor.

The elements of $\chi_{i j k, v}$ can be classified as nonmagnetic, i.e., $\quad \chi_{i j k, v}{ }^{(n m)}(\mathbf{M})=\chi_{i j k, v}{ }^{(n m)}(-\mathbf{M})$, or magnetic, i.e., $\chi_{i j k, v}{ }^{(m)}(\mathbf{M})=-\chi_{i j k, v}{ }^{(m)}(-\mathbf{M})$, susceptibility tensor elements. Similarly, the electric dipole moments can be separated into $\boldsymbol{\mu}_{v}^{(2 \omega)(m)}$ and $\boldsymbol{\mu}_{v}{ }^{(2 \omega)(n m)}$ magnetic and nonmagnetic emitted electric dipole contributions, respectively, which change or do not change their sign on reversal of the magnetic field. Selection rules for electric dipoles emitting at interfaces as a function of magnetization components are listed in Table 1. Unfortunately, even considering symmetry arguments, the too-large number of nonzero susceptibility tensor elements $\chi_{i j k, v}$ (see, e.g., Ref. 6) often prevents their experimental determination without crude assumptions. Other MSHG contributions can come from magnetization gradients ${ }^{16}$ or from antiferromagnetic order. ${ }^{6}$ The relevance of the two last-named contributions is discussed below.

Another limitation in the interpretation of MSHG data comes from difficulty in estimating the characteristic efficient thickness of the second-harmonic generation in the vicinity of a given interface from which second-harmonic light is emitted and that for all values of $\chi_{i j k, v}$. This effect has been discussed for particular situations. ${ }^{26,27}$

(iii) Radiating point dipoles $\boldsymbol{\mu}_{v}{ }^{(2 \omega)}$ on the $v$ th interface imply modified boundary conditions of the electric and magnetic fields at interfaces. ${ }^{24,28}$ The relationship between $\boldsymbol{\mu}_{v}{ }^{(2 \omega)}$ and outgoing electric field amplitudes $\epsilon_{\Pi, v}{ }^{(2 \omega)}$ at $2 \omega$ frequency (with $\Pi=\{s, p\}$ polarization states) is written as

\begin{tabular}{|c|c|c|c|c|}
\hline \multirow{2}{*}{$\begin{array}{l}\text { MSHG } \\
\text { Configuration }\end{array}$} & \multicolumn{4}{|c|}{ Type of Term } \\
\hline & Polar $M \| z$ & Longitudinal $M \| y$ & Transverse $M \| x$ & Nonmagnetic \\
\hline$p_{\text {in }} p_{\text {out }}$ & & & $\begin{array}{c}\mu_{y}=\chi_{y y y}{ }^{(m)} E_{y}{ }^{2}+\chi_{y z z}{ }^{(m)} E_{z}{ }^{2} \\
\mu_{z}=\underline{\chi_{z z y}{ }^{(m)} E_{y} E_{z}}\end{array}$ & $\begin{array}{c}\mu_{y}=\chi_{y z y}{ }^{(n m)} E_{z} E_{y} \\
\mu_{z}=\chi_{z y y}{ }^{(n m)} E_{y}{ }^{2}+\chi_{z z z}{ }^{(n m)} E_{z}{ }^{2}\end{array}$ \\
\hline $\begin{array}{l}p_{\text {in }} s_{\text {out }} \\
s_{\text {in }} p_{\text {out }} \\
s_{\text {in }} s_{\text {out }}\end{array}$ & $\mu_{x}=\underline{\chi_{x z y}{ }^{(m)} E_{y} E_{z}}$ & $\begin{array}{c}\mu_{x}=\frac{\chi_{x y y}{ }^{(m)} E_{y}{ }^{2}}{}+\chi_{x z z}{ }^{(m)} E_{z}{ }^{2} \\
\mu_{x}=\chi_{x x x}{ }^{(m)} E_{x}{ }^{2}\end{array}$ & $\mu_{y}=\overline{\chi_{y x x}{ }^{(m)} E_{x}^{2}}$ & $\mu_{z}=\chi_{z x x}^{(n m)} E_{x}^{2}$ \\
\hline
\end{tabular}

Table 1. Selection Rules for Magnetic and Nonmagnetic Terms ${ }^{\text {a }}$

${ }^{a}$ Components of the SHG radiated dipole moment $\boldsymbol{\mu}_{v}{ }^{(2 \omega)}$ generated by electric field $\mathbf{E}_{v}^{(\omega)}$ at the $v$ th interface for various MSHG configurations and interface magnetization components. To simplify the notation, we skip the superscripts $2 \omega$ for $\boldsymbol{\mu}_{v}{ }^{(2 \omega)}$ and $\omega$ for $\mathbf{E}_{v}{ }^{(\omega)}$. The underlined contributions are dominant. 


$$
\left[\begin{array}{c}
\epsilon_{s, v}{ }^{(2 \omega)} \\
\epsilon_{p, v}(2 \omega)
\end{array}\right]=\left[\begin{array}{ccc}
Z_{s x, v}{ }^{(2 \omega)} & 0 & 0 \\
0 & Z_{p y, v}{ }^{(2 \omega)} & Z_{p z, v}{ }^{(2 \omega)}
\end{array}\right]\left[\begin{array}{l}
\mu_{x, v}{ }^{(2 \omega)} \\
\mu_{y, v}{ }^{(2 \omega)} \\
\mu_{z, v}(2 \omega)
\end{array}\right],
$$

where $Z_{v}{ }^{(2 \omega)}$ stands for the matrix of generalized outgoing Fresnel elements.

(iv) The resultant outgoing electric field amplitudes, $\epsilon_{\Pi, \text { tot }}{ }^{(2 \omega)}$, of the entire multilayer structure (denoted by the subscript tot) are then determined first, by integration over all radiating dipoles located on each interface, and second, by summing of all interface contributions. When the magnetization and $\chi_{v}$ are uniform on each interface, $\epsilon_{\Pi, \text { tot }}{ }^{(2 \omega)}$ is simply expressed by a sum over $\epsilon_{\Pi, v}{ }^{(2 \omega)}$ originating from one point dipole on a given interface ${ }^{24}$.

$$
\epsilon_{\Pi, \mathrm{tot}}^{(2 \omega)}=\sum_{v} \epsilon_{\Pi, v}^{(2 \omega)}
$$

(v) The measured far-field second-harmonic generation radiated intensity is then given by ${ }^{24}$

$$
I_{\text {tot }}^{(2 \omega)} \sim\left|N_{z}^{(2 \omega)}\right|^{2}\left[\left|\epsilon_{s, \text { tot }}^{(2 \omega)}\right|^{2}+\left|\epsilon_{p, \text { tot }}^{(2 \omega)}\right|^{2}\right],
$$

where $N_{z}^{(2 \omega)}=\left\{\left[\mathrm{N}^{(2 \omega)}\right]^{2}-\left[N^{(\omega)} \sin \varphi\right]\right\}^{2}$, where $\varphi$ is the incidence angle. $N^{(\omega)}$ and $N^{(2 \omega)}$ are the refractive indices of the air at frequencies $\omega$ and $2 \omega$, respectively.

For different optical configurations the total magnetic emitted light intensity, $I_{\Pi, \text { tot }}{ }^{(2 \omega)(\mathrm{m})}$, that is linear in magnetization is

$$
I_{\Pi, \text { tot }}^{(2 \omega)(\mathrm{m})} \sim 2\left|N_{z}^{(2 \omega)}\right|^{2} \sum_{v} \mathfrak{R}\left\{\epsilon_{\Pi, v}{ }^{(2 \omega)(m)}\left[\epsilon_{\Pi, \text { tot }}{ }^{(2 \omega)(\mathrm{nm})}\right]^{\dagger}\right\},
$$

where the summation runs over all interfaces $v$ and ${ }^{\dagger}$ is a complex-conjugate symbol. $\quad \epsilon_{\Pi, \text { tot }}{ }^{(2 \omega)(n m)}=\Sigma_{v} \epsilon_{\Pi, v}(2 \omega)(n m)$ expresses the total nonmagnetic electric amplitude emitted by all interfaces.

Relation (6) shows that the magnetic part of the total radiated light intensity, $I_{\Pi, \text { tot }}(2 \omega)(m)$, is given simply by a summation over contributions from all interfaces. Note that the contribution that is due to the $v$ th interface, and proportional to the magnetization, is determined as the product of the magnetic part of the electric field radiated at the $v$ th interface and the total nonmagnetic part of the electrical field originating from all interfaces. As follows from relation $(6), I_{\Pi \text {, tot }}{ }^{(2 \omega)(m)}$ is related to products of nonlinear nonmagnetic and magnetic susceptibility elements, ${ }^{6} \chi_{v}{ }^{(n m)} \chi_{v}{ }^{(m)}$. The components of the radiating dipole $\boldsymbol{\mu}_{v}{ }^{(2 \omega)}$ are dependent only on particular components of the electric field, $\mathbf{E}_{v}{ }^{(\omega)}$ (Table 1).

In this section we have neglected the MSHG that is due to $\chi_{v}{ }^{(m)} \chi_{v}{ }^{(m)}$ products. This is justified because no quadratic contribution to magnetization has been evidenced in our case. As was mentioned previously, another possible mechanism can be associated with a nonuniform magnetization in-depth profile associated with the presence of a planar domain wall expanding inside the $\mathrm{Dy}_{30} \mathrm{Fe}_{58} \mathrm{Co}_{12}$ and $\mathrm{Fe}_{96} \mathrm{Si}_{4}$ layers. This can theoretically give rise to MSHG through a term proportional to the gra-

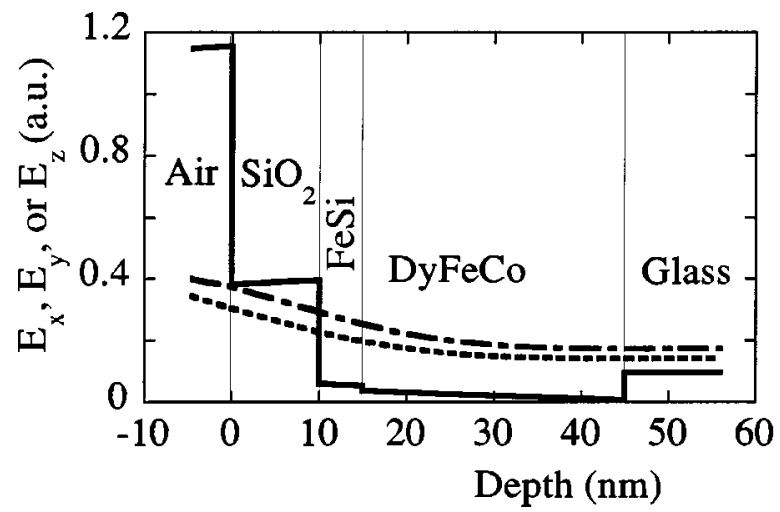

Fig. 3. In-depth profile of the amplitude of electric field components $\left|E_{z}{ }^{(\omega)}\right|$ (incident unitary $p$ wave, solid curve), $\left|E_{y}{ }^{(\omega)}\right|$ (incident unitary $p$ wave, dotted-dashed curve), and $\left|E_{y}{ }^{(\omega)}\right|$ (incident unitary $s$ wave, short-dashed curve) in the $\mathrm{SiO}_{2}(10-\mathrm{nm}) / \mathrm{Fe}_{96} \mathrm{Si}_{4}(5-\mathrm{nm}) / \mathrm{Dy}_{30} \mathrm{Fe}_{58} \mathrm{Co}_{12}(30-\mathrm{nm}) / \mathrm{glass}$ structure at a photon wavelength of $800 \mathrm{~nm}$ and for an incidence angle of $45^{\circ}$.

dient of the magnetization ${ }^{16}$ that may be nonzero inside a centrosymmetric medium, as in the layers themselves. Another contribution can come from the presence of antiferromagnetically aligned moments at the $\mathrm{Dy}_{30} \mathrm{Fe}_{58} \mathrm{Co}_{12} / \mathrm{Fe}_{96} \mathrm{Si}_{4}$ interface. ${ }^{6}$

The in-depth profile of the electric field inside the $\mathrm{Dy}_{30} \mathrm{Fe}_{58} \mathrm{Co}_{12} / \mathrm{Fe}_{96} \mathrm{Si}_{4}$ structure at frequency $\omega$ is presented in Fig. 3 for the $\mathrm{Fe}_{96} \mathrm{Si}_{4}(5-\mathrm{nm})$ film. The profile of the tangential electric field components, $E_{x}{ }^{(\omega)}$ and $E_{y}{ }^{(\omega)}$, decreases monotonically through the multilayer structure. In a counterpart, the profile of the normal component, $E_{z}{ }^{(\omega)}$, exhibits a steplike variation at interfaces. As discussed previously, ${ }^{15}$ the step amplitude is huge for metal-dielectric interfaces because they exhibit different diagonal permittivities, whereas it is rather small for the $\mathrm{Dy}_{30} \mathrm{Fe}_{58} \mathrm{Co}_{12} / \mathrm{Fe}_{96} \mathrm{Si}_{4}$ interface.

The calculated values of the generalized incoming Fresnel elements $X_{x s, v}{ }^{(\omega)}, X_{y p, v}{ }^{(\omega)}$, and $X_{z p, v}{ }^{(\omega)}$ are represented in polar form in Fig. 4. This presentation allows representation of the phase information on $\mathbf{E}^{(\omega)}$ at each interface. Assuming an incident field with normalized amplitude, the electric field modulus at the interfaces is given by $\left|E_{x}{ }^{(\omega)}\right|=\left|X_{x s, v}{ }^{(\omega)}\right|, \quad\left|E_{y}{ }^{(\omega)}\right|=\left|X_{y p, v}{ }^{(\omega)}\right|$, and $\left|E_{z}{ }^{(\omega)}\right|=\left|X_{z p, v}{ }^{(\omega)}\right|$. In as much as $E_{z}{ }^{(\omega)}$ is discontinuous at each interface, we define it as the average of $E_{z}{ }^{(\omega)}$ values at both sides of the interface. Penetrating further into the film causes the modules and the phases of $X_{x s, v}{ }^{(\omega)}$ and $X_{y p, v}{ }^{(\omega)}$ at the interfaces to decrease progressively. The largest value of $X_{z p, v}{ }^{(\omega)}$ is obviously obtained at the air- $\mathrm{SiO}_{2}$ interface, but it becomes negligible for the deeper $\mathrm{Fe}_{96} \mathrm{Si}_{4} / \mathrm{Dy}_{30} \mathrm{Fe}_{58} \mathrm{Co}_{12}$ and $\mathrm{Dy}_{30} \mathrm{Fe}_{58} \mathrm{Co}_{12}$ /glass interfaces.

The generalized outcoming Fresnel elements $Z_{s x, v}{ }^{(2 \omega)}$, $Z_{p y, v}{ }^{(2 \omega)}$, and $Z_{p z, v}{ }^{(2 \omega)}$ give access to the radiation emitted in the air by dipoles located on an interface $v$. They are represented in polar coordinates in Fig. 5 for our $\mathrm{Fe}_{96} \mathrm{Si}_{4}$ (5-nm) magnetic bilayer structure. The relation between the radiated intensity (in arbitrary units) and $Z_{\Pi i, v}{ }^{(2 \omega)}$ coefficients is written as $I_{S}=N_{0}{ }^{(2 \omega)}\left|N_{z, 0}{ }^{(2 \omega)} Z_{s x, v}{ }^{(2 \omega)}\right|^{2}, I_{P}$ $=N_{0}{ }^{(2 \omega)}\left|N_{z, 0}{ }^{(2 \omega)} Z_{p y, v}{ }^{(2 \omega)}\right|^{2}, \quad$ and $\quad I_{P}=N_{0}{ }^{(2 \omega)} \mid N_{z, 0}{ }^{(2 \omega)}$ $\times\left. Z_{p z, v}{ }^{(2 \omega)}\right|^{2}$, where $N_{0}{ }^{(2 \omega)}$ is the refractive index of air at frequency $2 \omega$ and $N_{z, 0}{ }^{(2 \omega)}$ is the $z$ component of the re- 
duced wave vector. These relations justify the representations in Fig. 5 of products such as $N_{z, 0}{ }^{(2 \omega)} Z_{\Pi i, v}{ }^{(2 \omega)}$ rather than $Z_{\Pi i, v}{ }^{(2 \omega)}$ alone. Both the modulus and the phase of $Z_{s x, v}{ }^{(2 \omega)}$ and $Z_{p y, v}{ }^{(2 \omega)}$ elements decrease continuously with the in-depth location of the interface. The situation is not so straightforward for the $Z_{p z, v}{ }^{(2 \omega)}$ ele-
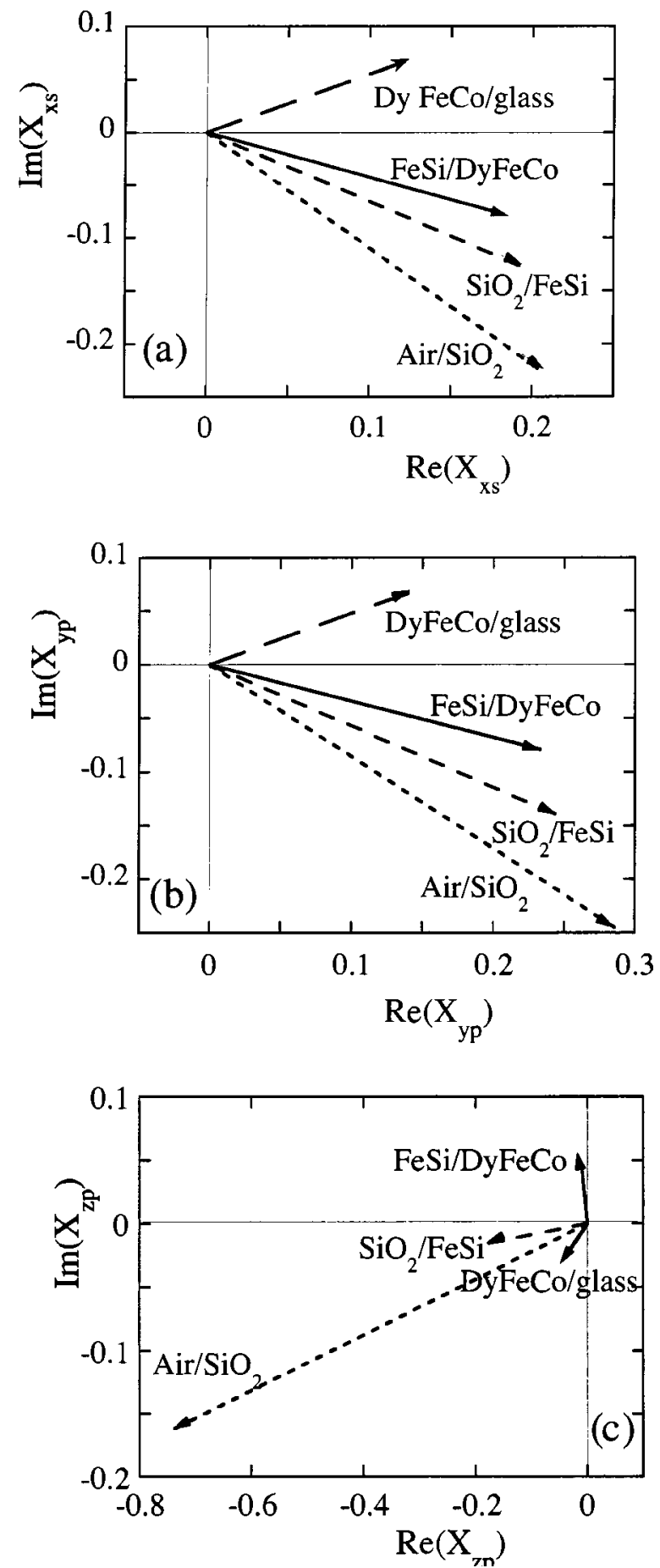

Fig. 4. Polar representation of the incoming Fresnel elements $X_{x s, v}{ }^{(\omega)}, X_{y p, v}{ }^{(\omega)}$, and $X_{z p, v}{ }^{(\omega)}$ for each interface $v$ for the $\quad \mathrm{SiO}_{2}(10-\mathrm{nm}) / \mathrm{Fe}_{96} \mathrm{Si}_{4}(5-\mathrm{nm}) / \mathrm{Dy}_{30} \mathrm{Fe}_{58} \mathrm{Co}_{12}(30-\mathrm{nm}) / \mathrm{glass}$ structure at a photon wavelength of $800 \mathrm{~nm}$ and for an incidence angle of $45^{\circ}$. The plotted quantity is $N_{z, 0}{ }^{(2 \omega)}$. Note that absolute values of $X$ are identical to those of the electric fields that are present on each interface as represented in Fig. 3.
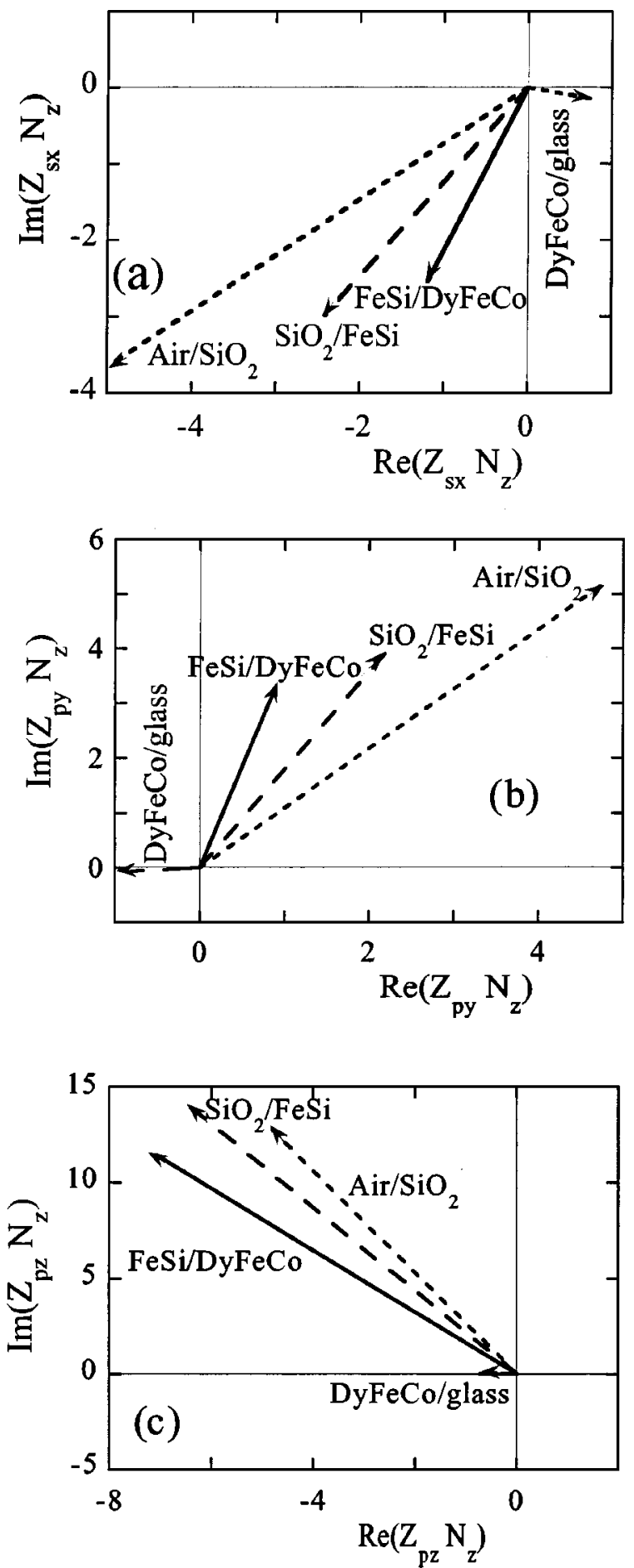

Fig. 5. Polar representation of the outgoing Fresnel elements $Z_{x s, v,-}{ }^{(2 \omega)}, Z_{y p, v,-}{ }^{(2 \omega)}$, and $Z_{z p, v,-}{ }^{(2 \omega)}$ coming from each interface $v$ for the $\mathrm{SiO}_{2}(10-\mathrm{nm}) / \mathrm{Fe}_{96} \mathrm{Si}_{4}(5-\mathrm{nm}) / \mathrm{Dy}_{30} \mathrm{Fe}_{58} \mathrm{Co}_{12}$ $\times(30-\mathrm{nm}) /$ glass structure at a photon wavelength of $400 \mathrm{~nm}$ and for light radiated at an angle of $45^{\circ}$. The light intensity is proportional to $\left|N_{z, 0}{ }^{(2 \omega)} Z_{i j, v,-}{ }^{(2 \omega)}\right|$, i.e., the modulus of the quantity plotted along the $x$ axis.

ments [i.e., related to the $\mu_{z, v}{ }^{(2 \omega)}$ dipole term], for which all contributions from the first three interfaces give nearly the same modulus and phase. The $Z_{p z, v}{ }^{(2 \omega)}$ element decreases more slowly with depth than do the $Z_{s x, v}{ }^{(2 \omega)}$ and $Z_{p y, v}{ }^{(2 \omega)}$ elements. 


\section{EXPERIMENTAL RESULTS AND DISCUSSION}

The LMOKE hysteresis loop, measured in the low field with light penetrating from the top film side (Fig. 1), is
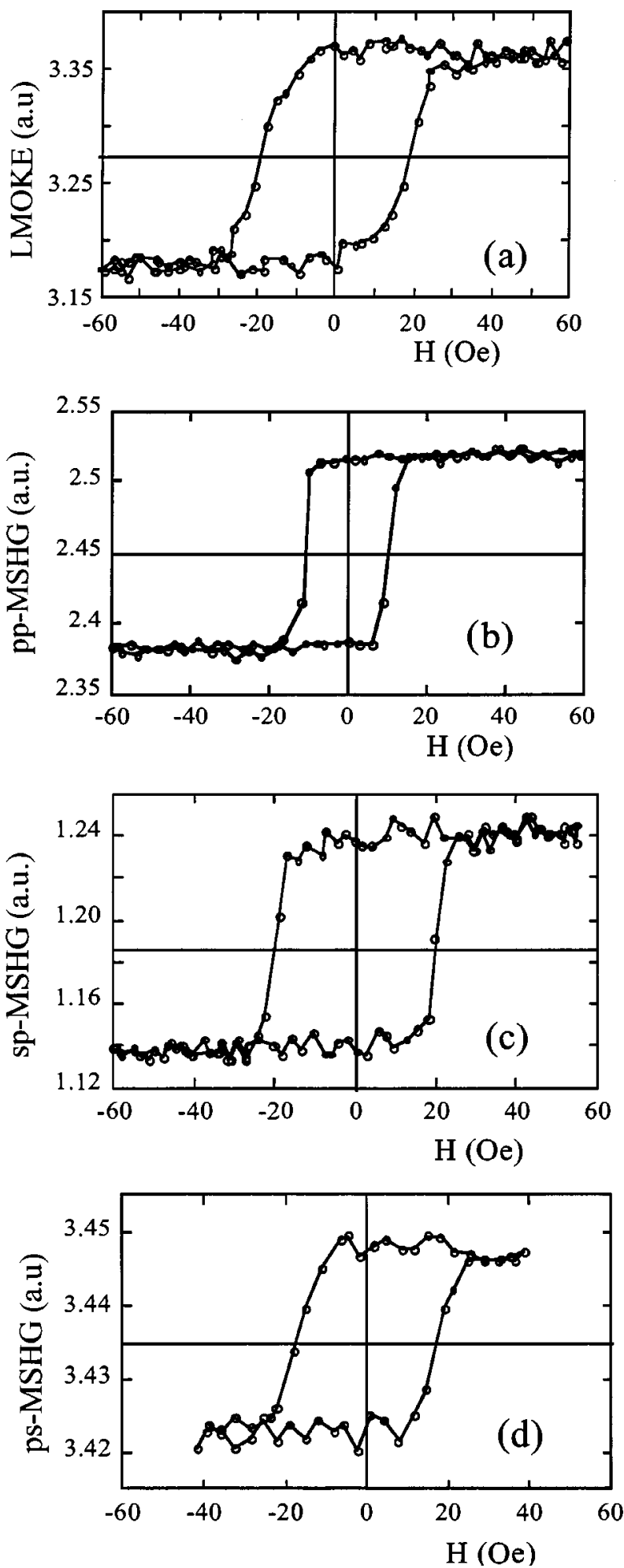

Fig. 6. MOKE and MSHG loops of the $\mathrm{Fe}_{96} \mathrm{Si}_{4}(5-\mathrm{nm}) / \mathrm{Dy}_{30} \mathrm{Fe}_{58} \mathrm{Co}_{12}$ structure measured in the same thermal conditions: (a) in the LMOKE, (b) in transverse $p p$ MSHG, (c) in transverse $s p$ MSHG, and (d) in longitudinal $p s$ MSHG.
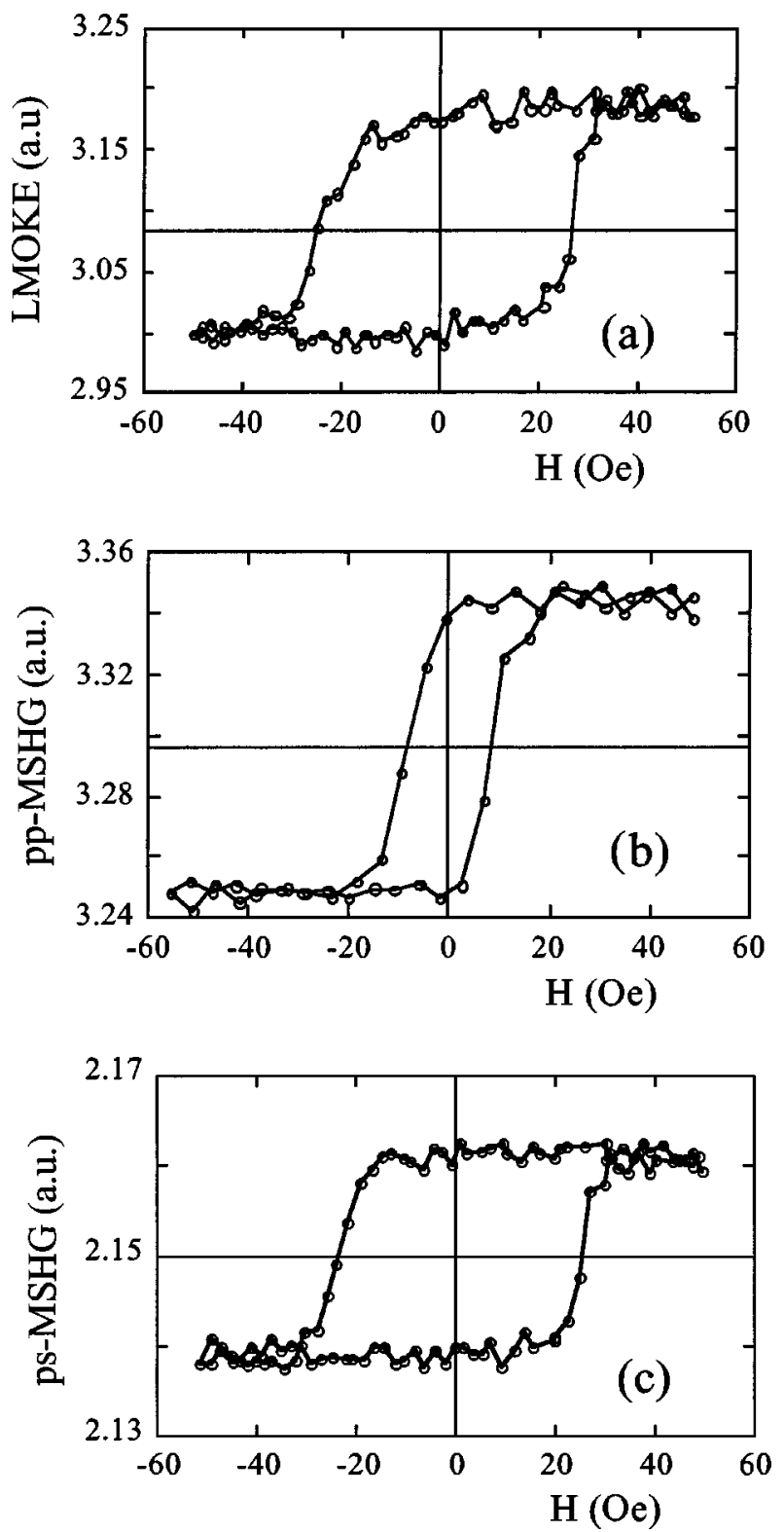

Fig. 7. MOKE and MSHG loops of the $\mathrm{Fe}_{96} \mathrm{Si}_{4}(10-\mathrm{nm}) / \mathrm{Dy}_{30} \mathrm{Fe}_{58} \mathrm{Co}_{12}$ structure measured in the same thermal conditions: (a) in the LMOKE, (b) in transverse $p p$ MSHG, and (c) in longitudinal ps MSHG.

essentially sensitive to the in-plane magnetic component of the $\mathrm{Fe}_{96} \mathrm{Si}_{4}$ layer. However, small and nearly reversible effects of a progressive rotation of $\mathrm{Dy}_{30} \mathrm{Fe}_{58} \mathrm{Co}_{12}$ and $\mathrm{Fe}_{96} \mathrm{Si}_{4}$ magnetization toward the in-plane field direction can be superimposed. LMOKE hysteresis loops, measured at $45^{\circ}$ of incidence, for $\mathrm{Fe}_{96} \mathrm{Si}_{4}$ (5- or 10-nm) films are shown in Figs. 6(a) and 7(a). The coercivity of the $\mathrm{Fe}_{96} \mathrm{Si}_{4}$ (5- or 10-nm) layers as measured with our MSHG setup is, respectively, $H_{c}=19$ and $H_{c}=25$ Oe. These values are reduced by a factor of $\sim 2$ as compared with those of coervivity measured by the LMOKE at room temperature under weak light. This effect is unambiguously due to local heating of the film by the rather intense laser beam spot. Independently knowing the variation of $H_{c}$ with temperature, we deduced that heating corresponds, in our case, to an elevation in temperature of $60^{\circ} \mathrm{C}$. This 
result is expected because the film is deposited on glass, which is an insulating material with low thermal conductivity. So, as claimed, inside the laser spot area the local temperature is raised to $\sim 80{ }^{\circ} \mathrm{C}$, i.e., close to the compensation temperature of $\mathrm{Dy}_{30} \mathrm{Fe}_{58} \mathrm{Co}_{12}$. Thus the $\mathrm{Dy}_{30} \mathrm{Fe}_{58} \mathrm{Co}_{12}$ layer exhibits a huge out-of-plane coercivity $(>20 \mathrm{kOe})$ at this temperature.

So the effective temperature of all MSHG measurements is $\sim 80^{\circ} \mathrm{C}$. Transverse $p p$ and $s p$ MSHG and longitudinal $p s$ MSHG hysteresis loops are still measured for light at $45^{\circ}$ incidence and for light penetrating the film structure from the top side (Fig. 1). Hysteresis loops for $\mathrm{Fe}_{96} \mathrm{Si}_{4}$ (5- or 10-nm) films are measured under a small inplane magnetic field [Figs. 6(b)-6(d), 7(b), and 7(c)]. For the two samples, the hysteresis loop obtained in transverse $p p$ MSHG is much more nearly square and exhibits a lower coercivity than the LMOKE, transverse $s p$ MSHG, or longitudinal $p s$ MSHG. This is true whatever the direction of the magnetization with respect to an inplane sample rotation. Moreover, LMOKE, longitudinal $p s$, and transverse $s p$ MSHG loop shapes are fairly different. This proves that all these effects have different in-depth sensitivities for probing the $\mathrm{Fe}_{96} \mathrm{Si}_{4}$ layer or interfaces.

Let us compare first the transverse $(\mathbf{H} \| \mathbf{x}) p p$ and $s p$ MSHG hysteresis loops for the $\mathrm{Fe}_{96} \mathrm{Si}_{4}$ (5-nm) film, measured at exactly the same place on the film surface [Figs. 6(b) and 6(c)]. The coercive field found in $p p$ configuration $(10 \mathrm{Oe})$ is significantly smaller than that determined in $s p$ configuration $(21 \mathrm{Oe})$; the latter value is close to that found in the LMOKE. Moreover, the $p p$ MSHG loop is much more nearly square. The difference in coercivity is explained as follows: $p p$ MSHG is presumably related more to spins located at the $\mathrm{SiO}_{2} / \mathrm{Fe}_{96} \mathrm{Si}_{4}$ interface, which are less closely coupled to any harder in-plane spin direction in the $\mathrm{Dy}_{30} \mathrm{Fe}_{58} \mathrm{Co}_{12}$ layer. Thus, during sweeping of the in-plane magnetic field, the spins begin to rotate at the top of the $\mathrm{Fe}_{96} \mathrm{Si}_{4}$ layer. This rotation generates a planar spiraling spin structure from the top to the inside of the $\mathrm{Fe}_{96} \mathrm{Si}_{4}$ layer. This expected magnetic behavior, coupled to our hysteresis loop observations, leads to the following points of experimental evidence:

(i) The transverse $p p$ MSHG is usually limited to probing the magnetization of the top $\mathrm{SiO}_{2} / \mathrm{Fe}_{96} \mathrm{Si}_{4}$ interface.

(ii) The transverse $s p$ MSHG is sensitive to both the first $\mathrm{SiO}_{2} / \mathrm{Fe}_{96} \mathrm{Si}_{4}$ and the second $\mathrm{Fe}_{96} \mathrm{Si}_{4} / \mathrm{Dy}_{30} \mathrm{Fe}_{58} \mathrm{Co}_{12}$ interfaces and certainly probes a region that spreads more widely about the interfaces.

Point (i) can be explained by the selection rules (Table 1) and by the estimation of the strength of the electric field at interfaces in $p p$ configuration. The $p$-polarized field at fundamental frequency $\omega$ contains both $E_{y}{ }^{(\omega)}$ and $E_{z}{ }^{(\omega)}$ components. The selectivity of $p p$ MSHG to the top $\mathrm{SiO}_{2}-\mathrm{Fe}_{96} \mathrm{Si}_{4}$ interface is associated with the large and abrupt jump of $E_{z}{ }^{(\omega)}$ at this interface compared with smaller changes at other magnetic interfaces (Fig. 3). The transverse $p p$ MSHG signal (Table 1) is related to $\mu_{y}{ }^{(2 \omega)}=\chi_{y y y}\left[E_{y}^{(\omega)}\right]^{2}+\chi_{y z z}\left[E_{z}^{(\omega)}\right]^{2}$ and $\mu_{z}^{(2 \omega)}=\chi_{z z y}$ $\times E_{y}{ }^{(\omega)} E_{z}{ }^{(\omega)}$. Hence the dominant term must be that related to $E_{z}{ }^{(\omega)}$, so $\chi_{y y y}$ becomes negligible compared with $\chi_{y z z}$ and $\chi_{z z y}$. Moreover, from a comparison of $p p$ and $s p$ MSHG loops, as we show below, the contribution of $\chi_{y z z}$ has to be small compared to that of $\chi_{z z y}$. In that picture the transverse $p p$ MSHG signal comes mainly from the $\mu_{z}{ }^{(2 \omega)}=\chi_{z z y} E_{y}{ }^{(\omega)} E_{z}{ }^{(\omega)}$ term. This conclusion is supported by the fact that the dipole radiation emitted by the $\mu_{z}^{(2 \omega)}$ component (and thus from $\chi_{z z y}$ ) is approximately three times more efficient than that which arises from $\mu_{y}{ }^{(2 \omega)}$ (Fig. 3). Furthermore, as was pointed out by Petukhov and Liebsch, ${ }^{27}$ and if one extends the results of $a b$ initio calculations obtained for $\mathrm{Al}(111)$ to our case, $\mu_{z}{ }^{(2 \omega)}$ must originate from a much thinner interface region $(\sim 0.1 \mathrm{~nm})$ than the two other components, $\mu_{x}{ }^{(2 \omega)}$ and $\mu_{y}{ }^{(2 \omega)}(\sim 1 \mathrm{~nm})$. This result supports the highly selective nature of the transverse $p p$ MSHG to the upper $\mathrm{SiO}_{2} / \mathrm{Fe}_{96} \mathrm{Si}_{4}$ interface, where there is a large contrast between diagonal permittivities. Similar results were indirectly found by Güdde et al. ${ }^{26}$ for ultrathin Co and $\mathrm{Ni}$ films deposited on $\mathrm{Cu}(001)$.

Point (ii) is consistent with the characteristic properties of the transverse sp MSHG. The incoming $s$-polarized field is associated with the $E_{x}{ }^{(\omega)}$ in-depth profile that keeps nearly the same value at both sides of the $\mathrm{SiO}_{2} / \mathrm{Fe}_{96} \mathrm{Si}_{4}$ or the $\mathrm{Fe}_{96} \mathrm{Si}_{4} / \mathrm{Dy}_{30} \mathrm{Fe}_{58} \mathrm{Co}_{12}$ interface (Fig. 3). The magnetic sp MSHG signal is then related to $\mu_{y}{ }^{(2 \omega)}=\chi_{y x x}\left[E_{x}^{(\omega)}\right]^{2}$ (Table 1). The intensity of the light radiated by the $\mu_{y}{ }^{(2 \omega)}$ dipole component is stronger by a factor 1.6 for the upper $\mathrm{SiO}_{2}-\mathrm{Fe}_{96} \mathrm{Si}_{4}$ interface than for the second interface (Fig. 5). Thus, if one assumes that $\chi_{y x x}$ is similar for the two interfaces, the two contributions must be efficient. This result is even better supported $^{27}$ by the fact that $\mu_{y}{ }^{(2 \omega)}$ probes a thicker region than $\mu_{z}{ }^{(2 \omega)}$.

As in transverse sp MSHG, the longitudinal $p s$ MSHG hysteresis loop exhibits a rather large coercivity, which means again that the second $\mathrm{Fe}_{96} \mathrm{Si}_{4}$ interface contributes more. Hence, the dominant term should not contain indepth selective $E_{z}{ }^{(\omega)}$ terms. As the term responsible for the magnetic signal in longitudinal $p s$ MSHG is written as $\mu_{x}{ }^{(2 \omega)}=\chi_{x y y}\left[E_{y}{ }^{(\omega)}\right]^{2}+\chi_{x z z}\left[E_{z}{ }^{(\omega)}\right]^{2}$ (Table 1), the contribution of $\chi_{x z z}\left[E_{z}^{(\omega)}\right]^{2}$ must be smaller than that which comes from $\chi_{x y y}\left[E_{y}{ }^{(\omega)}\right]^{2}$. This statement, associated with the symmetry relation, $\chi_{x z z}=-\chi_{y z z}$, was assumed in the discussion above of point (i), in which $\mu_{z}{ }^{(2 \omega)}$ $=\chi_{z z y} E_{y}{ }^{(\omega)} E_{z}{ }^{(\omega)}$ was the dominant term in the $p p$ configuration.

As was mentioned above, another MSHG contribution can come from antiferromagnetically aligned moments in $\mathrm{Dy}_{30} \mathrm{Fe}_{58} \mathrm{Co}_{12}$. Related MSHG effects have been predicted theoretically, ${ }^{6}$ but in our system they do not change sign with the reversal of the applied field. Considering the symmetry with respect to zero of recorded loops, we can exclude such a contribution.

Because of the nonuniform magnetization that exists inside the planar domain wall lying in the vicinity of the $\mathrm{Fe}_{96} \mathrm{Si}_{4} / \mathrm{Dy}_{30} \mathrm{Fe}_{58} \mathrm{Co}_{12}$ interface, one can expect a MSHG contribution related to magnetization gradients. ${ }^{16}$ Considering the symmetry of MSHG hysteresis loops, the related effect must reverse with the applied field. Thus, when $\mathbf{H} \| \mathbf{x}$, the $\mathrm{Fe}_{96} \mathrm{Si}_{4}$ magnetization has components along the $x$ or $y$ axis with $\Phi$-dependent amplitudes. Nearly no gradient MSHG terms are expected to come 
from the $\mathrm{Fe}_{96} \mathrm{Si}_{4} / \mathrm{SiO}_{2}$ interface because of the flat variation of the magnetization orientation in its vicinity. In counterpart, a gradient MSHG term can be generated in the neighborhood of the $\mathrm{Fe}_{96} \mathrm{Si}_{4} / \mathrm{Dy}_{30} \mathrm{Fe}_{58} \mathrm{Co}_{12}$ interface. In that case, considering the selection rules for MSHG gradient terms for an isotropic medium that are given in Table 1 of Ref. 16, only the local magnetization gradient in $\delta M_{x} / \delta z$ is found to be active in $p p$ and $s p$ polarization. It can give rise to several nonlinear susceptibility terms: $\chi_{y y y z X}, \chi_{z z z z X}, \chi_{z y z z X}$, and $\chi_{z z y z X}$ in $p p$ polarization and $\chi_{y x x z X}$ in $s p$ polarization. Again, as $p p$ MSHG shows a rather square hysteresis loop and a low coercivity, we can exclude here any gradient MSHG coming from the $\mathrm{Fe}_{96} \mathrm{Si}_{4} / \mathrm{Dy}_{30} \mathrm{Fe}_{58} \mathrm{Co}_{12}$ interface. The $s p$ MSHG can be generated by a gradient term, but its magnitude relative to the usual MSHG term, in proportion to the magnetization, cannot be determined.

MOKE and MSHG data obtained for $\mathrm{Fe}_{96} \mathrm{Si}_{4}$ 10- or 5 -nm film show rather similar magnetic behavior. This confirms all the above interpretations and proves that the optical phase changes introduced by different $\mathrm{Fe}_{96} \mathrm{Si}_{4}$ film thicknesses in the nanometer range do not have a large effect on the main features of the MSHG hysteresis loop.

\section{CONCLUSIONS}

We have presented evidence of highly selective $p p$ MSHG of $\mathrm{SiO}_{2} / \mathrm{Fe}_{96} \mathrm{Si}_{4}$ and $\mathrm{Fe}_{96} \mathrm{Si}_{4} / \mathrm{Dy}_{30} \mathrm{Fe}_{58} \mathrm{Co}_{12}$ exchange coupled bilayer structures and of transverse $s p$ MSHG and longitudinal $p$ s MSHG probing of the two $\mathrm{Fe}_{96} \mathrm{Si}_{4}$ interfaces with a greater selectivity. This explains why the two last hysteresis loops are comparable to those obtained in the LMOKE, which is known to probe in-depth magnetization more nearly uniformly. ${ }^{29}$ However, as expected, the loop becomes increasingly more rounded when one goes from transverse $p p$ to transverse $s p$ longitudinal $p s$ MSHG and LMOKE. One can interpret this result qualitatively by saying that sensitivity and selectivity to interfaces becomes correspondingly less pertinent.

As we know, the MSHG signal comes from the interference between nonlinear nonmagnetic and magnetic susceptibilities. In our case, when magnetization varies with depth in the structure essentially in the vicinity of the $\mathrm{Fe}_{96} \mathrm{Si}_{4}-\mathrm{Dy}_{30} \mathrm{Fe}_{58} \mathrm{Co}_{12}$ interface, we have demonstrated that only breaking of the nonmagnetic symmetry will reveal large MSHG signals. The most efficient factor that controls the MSHG in-depth selectivity is the steplike profile of the $z$ component of the electric field that becomes large at metal-insulator interfaces owing to the abrupt change of the diagonal permittivity tensor elements. Thus one can say that the magnitude of MSHG is related to the permittivity gradient at interfaces over the effective depth probed by second-harmonic generation. The most efficient terms that are responsible for a MSHG signal have been determined in each normal optical configuration. From hysteresis loop investigations it is unfortunately not possible to determine the magnitude of MSHG gradient terms related to the planar wall.

Finally, our results confirm again, as for the $\mathrm{CoO} / \mathrm{NiFe} /$ $\mathrm{NiO} / \mathrm{Co} / \mathrm{Cu}$ structure, ${ }^{5}$ that the $\mu_{z}{ }^{(2 \omega)}$ dipole component is emitted from a thinner region at interfaces than for $\mu_{x}{ }^{(2 \omega)}$ and $\mu_{y}{ }^{(2 \omega)}$. For this structure we have already proved that $p p$ MSHG is more selective at interfaces but in this case is generated from the third buried interface and not from the first one as for $\mathrm{Fe}_{96} \mathrm{Si}_{4} / \mathrm{Dy}_{30} \mathrm{Fe}_{58} \mathrm{Co}_{12}$. But, again, the metal-dielectric interface has to be considered the most efficient radiation source in the $p p$ configuration. As MSHG should be of great interest for testing tunnel junction interfaces in giant magnetoresistive devices, more experiments in simple multilayer structures need to be performed to confirm simple predictions of selectivity.

\section{ACKNOWLEDGMENTS}

The authors thank P. Malouin and G. Tessier for sharing results of their early MSHG experiments with this system. V. V. Pavlov has benefited from an associate researcher grant from the Center National de la Recherche Scientifique (France). L. Sampaio thanks the Conselho Nacional de Desenvolvimento Científíco e Technologico (Brazil) for funding his stay as a visiting scientist. The stay of J. Hamrle was partly funded by the Magnetism at Nanometer Scale: Experiment and Theory (contract HPMT-CT-2000-00066) European Union Marie-Curie project; he thanks S. Visnovsky, his Ph.D. thesis supervisor.

\section{J. Ferre's e-mail address is ferre@lps.u-psud.fr.}

*On leave from the Centro Brasiliero de Pesquinas Fisicas, Urca, Rio de Janeiro, Brazil.

On leave from the Quantum Nano-Scale Magnetic Laboratory, Frontier Research System, RIKEN, Saitama, Japan.

${ }^{\ddagger}$ On leave from the Ioffe Physico-Technical Institute, St. Petersburg, Russia.

\section{REFERENCES}

1. J. Ferré, "Linear and non-linear magneto-optical effects," in Magnetism and Synchrotron radiation, E. Beaurepaire, F. Scheurer, G. Krill, and J.-P. Kappler, eds., Vol. 565 of Lecture Notes in Physics (Springer-Verlag, Berlin, 2001), pp. 316-335.

2. R. Allenspach, "Spin-polarized scanning electron microscopy," IBM J. Res. Dev. 44, 553-570 (2000).

3. K. Grzelakowski, T. Duden, E. Bauer, H. Poppa, and S. Chiang, "A new surface microscope for magnetic imaging," IEEE Trans. Magn. 30, 4500-4502 (1994).

4. O. Pletzsch, A. Kubetzka, M. Bode, and R. Wiesendanger, "Observation of magnetic hysteresis at the nanometer scale by spin-polarized scanning tunneling spectroscopy," Science 292, 2053-2056 (2001).

5. R.-P. Pan, H. D. Wei, and Y. R. Shen, "Optical secondharmonic generation from magnetized surfaces," Phys. Rev. B 39, 1229-1234 (1989).

6. R.-H. Benneman, ed., Non-Linear Optics in Metals, Vol. 98 of International Series of Monographs on Physics (Oxford U. Press, Oxford, UK, 1998).

7. J. Reif, J. C. Zink, C.-M. Schneider, and J. Kirshner, "Effects of surface magnetism on optical second-harmonic generation," Phys. Rev. Lett. 67, 2878-2881 (1991).

8. H. A. Wieranga, W. de Jong, M. W. J. Prins, Th. Rasing, R. Vollmer, A. Kirilyuk, H. Schwabe, and J. Kirshner, "Interface magnetism and possible quantum well oscillations in ultrathin $\mathrm{Co} / \mathrm{Cu}$ films observed by magnetization-induced 
second harmonic generation,” Phys. Rev. Lett. 74, 1462 1465 (1995).

9. M. Straub, R. Vollmer, and J. Kirshner, "Surface magnetism of ultrathin $\gamma$-Fe films investigated by nonlinear magnetooptical Kerr effect," Phys. Rev. Lett. 77, 743-746 (1996).

10. A. Kirilyuk, "Nonlinear optics in application to magnetic surfaces and thin films," J. Phys. D 35, R189-R207 (2002).

11. G. Spiering, V. Koustos, H. Wierenga, M. Prins, D. Abraham, and T. Rasing, "Interface magnetism studied by optical second harmonic generation," J. Magn. Magn. Mater. 121, 109-111 (1993).

12. Y. Z. Wu, R. Vollmer, H. Regensburger, X.-F. Jin, and J. Kirshner, "Magnetization-induced second harmonic generation from the $\mathrm{Ni} / \mathrm{Cu}$ interface in multilayers on $\mathrm{Cu}(001)$," Phys. Rev. B 63, 054401 (2001).

13. A. Kirilyuk, Th. Rasing, M. A. M. Haast, and J. C. Lodder, "Probing structure and magnetism of $\mathrm{CoNi} / \mathrm{Pt}$ interfaces by nonlinear magneto-optics,” Appl. Phys. Lett. 72, 2331-2333 (1998).

14. L. C. Sampaio, A. Mougin, J. Ferré, P. Georges, A. Brun, H. Bernas, S. Poppe, T. Mewes, J. Fassbender, and B. Hillebrands, "Probing interface magnetism in the $\mathrm{FeMn} / \mathrm{NiFe}$ exchange bias system using magnetic second harmonic generation," Europhys. Lett. 63, 819-825 (2003).

15. L. C. Sampaio, J. Hamrle, A. Mougin, J. Ferré, F. Garcia, F. Fettar, B. Dieny, and A. Brun, "In-depth sensitivity and magnetic second-harmonic generation of light in a multilayer structure," Phys. Rev. B 70, 104403 (2004).

16. A. V. Petukhov, I. L. Lyubchanskii, and Th. Rasing, "Theory of nonlinear magneto-optical imaging of magnetic domains and domain walls," Phys. Rev. B 56, 2680-2687 (1997).

17. T. Kobayashi, H. Tsuji, S. Tsunashima, and S. Uchiyama, "Magnetization process of exchange-coupled ferrimagnetic double-layered films," Jpn. J. Appl. Phys., 20, 2089-2095 (1981).

18. N. Nishimura and S. Tsunashima, "MSR media using inplane magnetization layers without a readout bias magnet," J. Magn. Soc. Jpn. 20, 97-102 (1996).
19. X. Hu and Y. Kawazoe, "Theory of the capping effect in magnetic double-film systems," Phys. Rev. B 49, 3294-3302 (1994).

20. O. Redon and P. P. Freitas, "Mechanism of exchange anisotropy and thermal stability of spin valves biased with ultrathin TbCo layers,” J. Appl. Phys. 83, 2851-2856 (1998).

21. M. J. Donahue and D. G. Porter, "OOMMF Users' Guide, version 1.0,” NIST interagency rep. NISTIR 6376 (National Institute of Standards and Technology, Gaithersburg, Md., 1999) (http://math.nist.gov/oommf).

22. R. Sbiaa, H. Le Gall, O. Koshkina, and M. El Harfaoui, "Theoretical investigation of temperature induced phase transition in exchange coupled double layers," J. Appl. Phys. 81, 5236-5238 (1997).

23. V. V. Pavlov, J. Ferré, P. Meyer, G. Tessier, P. Georges, A. Brun, P. Beauvillain, and V. Mathet, "Linear and nonlinear magneto-optical studies of $\mathrm{Pt} / \mathrm{Co} / \mathrm{Pt}$ thin films," J. Phys. Condens. Matter 13, 9867-9878 (2001).

24. J. Hamrle, L. Polerecky, and J. Ferré, "Theory of secondharmonic generation from multilayer systems based on electric point-dipole radiation: application to magnetic multilayers," Phys. Rev. B 68, 144401 (2003).

25. H. A. Wierenga, M. W. J. Prins, and Th. Rasing, "Magnetization-induced optical second harmonic generation from magnetic multilayers," Physica B 204, 281-286 (1995).

26. J. Güdde, J. Hohlfeld, and E. Matthias, "About the depth sensitivity of second-harmonic radiation in ultra-thin metal films," Appl. Phys. B 74, 691-695 (2002).

27. A. Petukhov and A. Liebsch, "Surface anisotropy in optical second harmonic generation. I. $\mathrm{Al}(111)$," Surf. Sci. 334 195-208 (1995).

28. R. Atkinson and N. F. Kubrakov, "Boundary conditions in the simplest model of linear and second harmonic magnetooptical effects," Phys. Rev. B 65, 014432 (2002).

29. J. Hamrle, J. Ferré, M. Nyvlt, and S. Visnovsky, "In-depth resolution of the magneto-optical Kerr effect in ferromagnetic multilayers," Phys. Rev. B 66, 224423 (2002). 